\title{
Editorial
}

\section{Gift authorships and research awards}

H.C.T.S. Abeysena ${ }^{1}$

Authorship is the fulfillment of the responsibility to communicate research results to the scientific community for external evaluation and the primary basis for assessing a scientist's contributions to developing new knowledge (1). It is also a way of giving credit for intellectual work. Research publications are also important for academic promotions, when applying for better positions elsewhere and acquiring research grants. Further, it adds to the reputation of an individual as well as to the institution the person is attached to, as the quality of academic institutions are evaluated on part by publications. In addition it recognizes the expertness in research methods, integrity of the work and productivity.

International Committee of Medical Journal Editors (ICMJE) has developed standards for publication of papers in biomedical journals and produced its first set of authorship guidelines, entitled "Uniform Requirements for Manuscripts Submitted to Biomedical Journals", in 1979. According to the latest version of the guidelines (2) the authorship credit should be based on "1) substantial contributions to conception or design of the work or acquisition, analysis and interpretation of data, 2) drafting the article or revising it critically for important intellectual content, and 3) final approval of the version to be published. and 4) agreement to be accountable for all aspects of the work in ensuring that questions related to the accuracy or integrity of any part of the work are appropriately investigated and resolved". Authors should meet all the four conditions above to be eligible for authorship. The fourth condition reflects the extent of responsibility demonstrated by authors regarding the content included in the paper. If a person has contributed substantially for a paper, then he/she should be able to take responsibility and explain or comment on any concerns raised by the reviewers or editors. Failure to do so is an indication of promiscuous authorship.

It is widely accepted that the first author is the person who has carried out a majority of the research work described in the paper. The MD or PhD students are obviously required to perform the bulk of the work in their thesis, and they must contribute substantially to the intellectual development of the manuscript. Therefore the student must be the first author of almost all the articles published from the thesis. Senior author is placed at last on an authorship list. Senior authorship is defined as individuals who generally direct, oversee, and guarantee the authenticity of the work reported and "implicitly take responsibility for the work's scientific accuracy, valid methodology, analysis, and conclusions. Senior author is to take responsibility for the research as a whole and generally be the last author" (3).

Based on afore mentioned authorship criteria, it is evident that acquisition of funding, collection of data, or general supervision of the research groups alone, do not justify authorship. Further searching literature for the research, offering validated questionnaires for data collection, provision of recruitment of patients/specimens or allowing ward/clinic settings for patient recruitment, offering reagents or laboratory facilities for biochemical, microbiological or pathological analysis and providing digital or technological assistance such as instruments/hardware/software for data collection or analyses are not in themselves sufficient contributions to justify authorship. Individuals who have made valuable contribution to a publication; for example by reviewing or approving or even writing a manuscript

\footnotetext{
1. Professor in Public Health, Faculty of Medicine, University of Kelaniya, Sri Lanka

*Correspondence: chrishantha-abeysena@kln.ac.lk

DOI: $10.4038 /$ jccpsl.v22i1.8081
} 
before submission, providing care and supervising or recruiting data collectors, but who do not meet the criteria for authorship, should ideally be acknowledged.

Honorary or gift authorships are defined as the awarding of authorship to those who do not contribute substantially for the intellectual work out of respect or friendship, with a hope of mutual benefits and/ or to give the paper a greater sense of legitimacy $(4,5)$. Some investigators offer authorship for the department chair or head, dean or a director of an academic or health institution, where as in some instances they demand the authorship by way of inducement. It is not uncommon to find departments wherein there is an unwritten rule that the name of the head of the department (or head of the clinical unit) has to be included in every paper. Some senior colleagues are extremely ambitious, selfish and insincere, that efforts are made to sabotage having an honest and authentic author list, despite being well aware of the ICJME guidelines. Hence one of their strategies is to demonstrate a public involvement, and there by simulate to conform to authorship criteria, by discussing the research work at scientific and public forums. Mutual support authorships have been defined as an agreement by two or more investigators to place their names on each other's papers to give the appearance of higher productivity (4). Mutual support may be in terms of covering routine teaching activities of the other person or duties in provision of patient or health care by colleagues. Offering authorship to the spouse is also another form of gift authorship.

In the Sri Lankan context, there are several forms of incentives offered to motivate individuals in to publishing research articles. Eligibility to claim for the research allowance paid on a monthly basis both by universities and Ministry of Health, bestowing with annual presidential awards and computation of citation indices (H index) which measures productivity and citation impact are some of these inducements. All above would enhance authors' image as eminent researchers. One of the convenient methods to reach the above targets is by offering/accepting gift authorships, which could abruptly multiply the number of publications. This will also ensure that the same authors will remain in the higher ranks year in and year out. As for the scientific content of a paper, publishing with unwarranted co-authorships should be viewed as a form of deception resulting in professional misconduct (6).

The prevalence of guest/honorary authorship is reported to range between $11-60 \%(7,8)$, which is certainly an underestimate. It is also reported that articles with $>5$ authors are more likely to have "honorary authors" than those with $\leq 3$ authors (7). However one of the reasons for having a large number of authors per article could be explained on the basis of complexity of research conducted in biomedical arena.

Certain mechanisms against honorary authorships has been implemented by some scientific journals. Inclusion of a statement which specifies the contribution of every author is one such measure. However the possibility of a person making vague claims for authorship cannot be totally eliminated. Trustworthiness of authorship claims mainly depend on an individual's values and ethical behavior.

The detection of gift authorships is a difficult task. However it is possible to predict gift authorship based on the following criteria: 1) more publications per year with a long list of authors, 2) being the last author of the list, 3) inclusion of spouse's name in several publications, 4) holding administrative posts over several years or higher posts in several institutions simultaneously, 5) lack of formal education on epidemiology and biostatistics, and 6) general ethical and professional behaviour of the person. 
Therefore, the expertness and credibility of scientific research and scientific publications should not be based on the number of publications or number of awards earned by an individual in the context of current socio-cultural and ethical dilemma.

\section{References}

1. National Institute of Health. Guidelines for the Conduct of Research in the Intramural Research Program at NIH [Internet]. National Institutes of Health, Bethesda, MD, 2007. http://sourcebook. od.nih.gov/ethic-conduct/Conduct\%20Research\%206-11-07.pdf.

2. International Committee of Medical Journal Editors. Recommendations for the conduct, reporting, editing, and publication of scholarly work in medical journals [Internet]. International Committee of Medical Journal Editors, Philadelphia, PA, 2013. http://www.icmje.org/.

3. McKneally M. Put my name on that paper: reflections on the ethics of authorship. J Thorac Cardiovasc Surg 2006; 131: 517-519.

4. Claxton LD. Scientific authorship. Part 2. History, recurring issues, practices, and guidelines. Mutat Res 2005; 589: 31-45.

5. Flanagin A, Carey LA, Fontanarosa PB, Phillips SG, Pace BP, Lundberg GD, Rennie D. Prevalence of articles with honorary authors and ghost authors in peer-reviewed medical journals. JAMA 1998; 280: 222-224.

6. Strange, K. Authorship: why not just toss a coin? Am J Physiol Cell Physiol. 2008; 295: C567C575.

7. Slone RM. Coauthors' contributions to major papers published in the AJR: frequency of undeserved coauthorship. AJR Am J Roentgenol. 1996;167:571-9.

8. Kressel HY, Dixon AK. Where is the Honor in Honorary Authorship? Radiology 2011;259:324-7.

Editorial Office:

Email: editorjccpsl@gmail.com

The College of Community Physicians of Sri Lanka, No. 06, "Wijerama House", Wijerama Mawatha, Colombo 07, Sri Lanka. 